\title{
Trapped Field Analysis of a High Temperature Superconducting Bulk with Artificial Holes
}

\author{
Guneik Jang ${ }^{1 *}$, Mansoo Lee ${ }^{1}$, Seungyong Han ${ }^{2}$, Chanjoong Kim³ ${ }^{3}$ Younghee Han ${ }^{4}$, and Byungjoon Park ${ }^{4}$ \\ ${ }^{1}$ Department of Advanced Materials Engineering, Chungbuk National University, Cheongju 361-763, Korea \\ ${ }^{2}$ Francic Bitter Magnet Laboratory, Massachusetts Institute of Technology, Cambridge, MA 02193, USA \\ ${ }^{3}$ Superconductor Laboratory, Korea Atomic Energy Research Institute, Daejeon 305-380, Korea \\ ${ }^{4}$ Green Growth Laboratory, Korea Electric Power Research Institute, Daejeon 305-760, Korea
}

(Received 25 March 2011, Received in final form 22 April 2011, Accepted 25 April 2011)

\begin{abstract}
To improve trapped field characteristics of a high temperature superconducting (HTS) bulk, a technique to implement artificial holes has been studied. The artificial holes, filled up with epoxy or metal, may provide better cooling channel and enhance mechanical strength of the HTS bulk. Although many useful researches based on experiments have been reported, a numerical approach is still limited because of several reasons that include: 1) highly non-linear electromagnetic properties of HTS; and 2) difficulty in modeling of randomly scattered "small" artificial holes. In this paper, a 2-D finite element method with iteration is adopted to analyze trapped field characteristics of HTS bulk with artificial holes. The validity of the calculation is verified by comparison between measurement and calculation of a trapped field in a $40 \times 40 \mathrm{~mm}$ square and $3.1 \mathrm{~mm}$ thick HTS bulk having 16 artificial holes with diameter of $0.7 \mathrm{~mm}$. The effects of sizes and array patterns of artificial holes on distribution of trapped field within HTS bulk are numerically investigated using suggested method.
\end{abstract}

Keywords : artificial hole, finite element method, high temperature superconducting bulk, levitation force, trapped field

\section{Introduction}

The melt textured $\mathrm{YBa}_{2} \mathrm{Cu}_{3} \mathrm{O}_{7-\delta}(\mathrm{YBCO})$ bulk is an attractive material for a bearing part of superconducting flywheel energy storage system (SFES) due to the high levitation force and the trapped magnetic field properties. It has been known that bulk YBCO containing internal defects such as gas pores and cracks have caused the failure of a superconducting bulk owing to the electromagnetic force induced by a trapped magnetic field. Therefore a number of researches have been carried out to improve the mechanical properties of melt textured YBCO. Recently it has been proposed to drill arrays of artificial holes within high temperature superconducting bulk in order to improve their chemical and thermal properties [16]. The results of the researches have proved that the artificial holes prevented the fracture of HTS bulk by thermal and magnetic stress during cool-down or magnetization. The artificial holes with larger interfacial area and filled with epoxy or metal, may provide better cooling

\footnotetext{
*Corresponding author: Tel: +82-43-261-2412
}

Fax: +82-43-271-3222, e-mail: gejang@chungbuk.ac.kr channel and also enhance mechanical strength of the HTS bulk.

However, most researches regarding the artificial holes have been based on experiments; numerical approaches [6] may be limited by several reasons such as: 1) highly non-linear electric properties of HTS bulk; 2) difficulty in modeling of scattered "small" artificial holes. Although a 3-D finite element method (FEM) will be required for precise modeling of scattered artificial holes [7-9], a 2-D FEM is adopted to save time during iterative calculation to consider non-linearity of HTS material because the volume of a hole is $\sim 4,000$ times smaller than that of the bulk and also too many elements need to be generated in the 3-D FEM. This paper presents a fast 2-D computational approach to estimate trapped field variations in an HTS bulk before and after artificial holes are implemented. The validity of the suggested method is verified by comparison between measurement and calculation of a trapped field in an HTS bulk with 16 holes with diameter of $0.7 \mathrm{~mm}$. The effects of sizes and patterns of artificial holes on trapped field distribution are investigated by suggested method. 


\section{Numerical Analysis}

\subsection{Governing equation}

Governing equation with magnetic vector potential, $\vec{A}=\left(A_{x}, A_{y}\right)$, is presented in (1)

$$
\sigma \mu \frac{\partial \vec{A}}{\partial t}+\vec{\nabla} \times(\vec{\nabla} \times \vec{A})=\mu \vec{J}
$$

where $\sigma, \mu$, and $\vec{J}$ are electrical conductivity, magnetic permeability, and current density, respectively.

To consider the limited length of an HTS bulk in the 2-

D FEM, a calibration factor, $\gamma_{\mathrm{c}}$, is adopted as (2)

$$
\gamma_{c}=B_{3 D} / B_{2 D}
$$

where $B_{3 D}$ and $B_{2 D}$ are axial magnetic fields at the center of a permanent magnet, of which dimensions are identical to those of the target HTS bulk, calculated by 3D (actual dimension) and 2D (infinite long) FEM, respectively. Then, the axial magnetic field calculated by the present 2 D FEM is multiplied by the calibration factor to estimate the actual field.

\subsection{E-J constitute relation}

In the 2-D FEM, currents flowing only in the a-b plane are considered while those in the a-c and b-c plane are assumed to be negligible; only the axial field is taken into consideration. To numerically consider the non-linear electrical conductivity of an HTS bulk, a critical state model with iteration was adopted as equation (3) and (4)

$$
\begin{aligned}
& \sigma^{i+1}(E)=\frac{J_{c}}{E}=\sigma^{i}(E) \frac{J_{c}}{J^{i}} \quad \text { if } J^{i}>J_{c} \\
& \sigma^{i+1}(E)=\sigma^{i}(E) \quad \text { if } J^{i} \leq J_{c}
\end{aligned}
$$

where $J_{c}$ is critical current density of an HTS bulk and $\sigma_{i}$ and $J^{i}$ are electrical conductivity and current density of each element in the $i$-th iteration [10].

For more accurate simulation, the Kim model, as seen in equation (5), is used for the $J_{c}$ in equations (3) and (4)

$$
J_{c}(B)=J_{c o} \frac{B_{c o}}{|B|+B_{c o}}
$$

where $J_{c o}$ and $B_{c o}$ are critical current density at zero field and reference magnetic field to make the critical density as the half of $J_{c o}$, respectively.

\subsection{Validity of the suggest numerical approach}

With the suggested method, a trapped field of an YBCO bulk with 16 artificial holes was calculated and compared with the actual measured one. Table 1 presents a summary of the YBCO bulk parameters. Fig. 1 shows a picture of
Table 1. Parameters of the YBCO bulk.

\begin{tabular}{lrc}
\hline \hline Parameters & & Values \\
\hline HTS Bulk & & \\
$\quad$ Material & YBCO \\
Width (square) & {$[\mathrm{mm}]$} & $40 \times 40$ \\
Thickness & {$[\mathrm{mm}]$} & 3.1 \\
$\mathrm{~J}_{\mathrm{co}}$ (measured) & {$\left[\mathrm{MA} / \mathrm{cm}^{2}\right]$} & 0.51 \\
$\mathrm{~B}_{\mathrm{co}}$ & {$[\mathrm{T}]$} & 0.25 \\
$\quad$ End effect calibration factor, $\gamma_{\mathrm{c}}$ & {$[\mathrm{null}]$} & 0.208 \\
$\begin{array}{l}\text { Artificial hole } \\
\text { Number of holes }\end{array}$ & & \\
Hole diameter & {$[\mathrm{mm}]$} & 0.7 \\
$\begin{array}{l}\text { Distance between holes in x-y direction } \\
\text { between adjacent holes }\end{array}$ & {$[\mathrm{mm}]$} & 8.0 \\
\hline
\end{tabular}

the YBCO bulk.

The 16 artificial holes, $0.7 \mathrm{~mm}$ in diameter, were mechanically drilled as seen in Fig. 1. The distance between holes is $8 \mathrm{~mm}$ in $\mathrm{X}$ and $\mathrm{Y}$ direction. A $4040 \mathrm{~mm} \mathrm{Nd-Fe-B}$ permanent magnet with surface flux density of $0.5 \mathrm{~T}$ was used for trapping field into the HTS bulk in of liquid nitrogen at $77 \mathrm{~K}$ by field cooling process. Since the field from the permanent magnet was much larger than the maximum trapped field of the HTS bulk, $0.195 \mathrm{~T}$, the induced current in the bulk was fully saturated in the a-b plane along the c-axis according to the critical state model. Thus, in the 2-D FEM simulation, the permanent magnet was modeled as an equivalent solenoid electromagnet to apply enough axial fields for maximum field cooling.

Fig. 2 shows a) measured axial field, b) calculated axial field, and c) calculated current distribution on the top surface of the YBCO test bulk; the peak field at center was calculated $0.194 \mathrm{~T}$ which was similar to the measured one, $0.195 \mathrm{~T}$. Although the induced currents bypassed

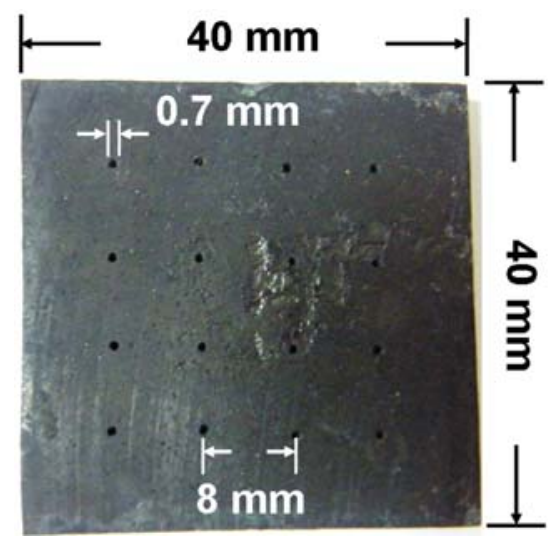

Fig. 1. Picture of an YBCO bulk with 16 artificial holes; YBCO bulk: $40 \times 40 \mathrm{~mm}$ square and $3.1 \mathrm{~mm}$ thick; diameter of each hole: $0.7 \mathrm{~mm}$. 
the artificial holes in Fig. 2c, the peak field was barely affected by the presence of artificial holes because the volume occupied by hole is negligible to that of the total HTS bulk. The measured (Fig. 2a) and calculated (Fig. 2b) field distributions are slightly different in details

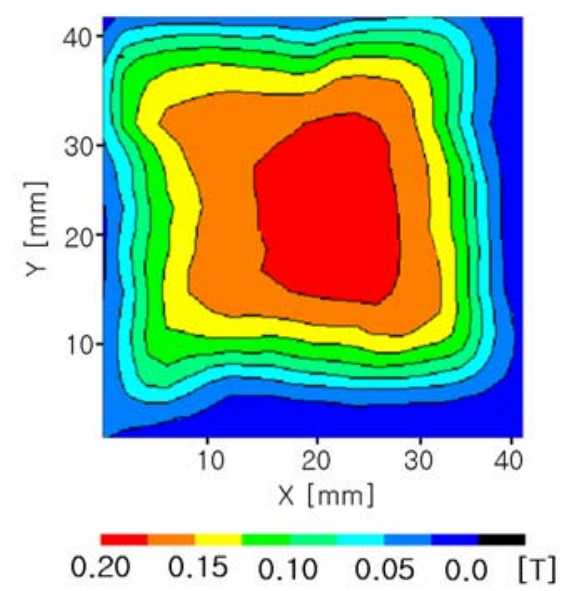

(a)

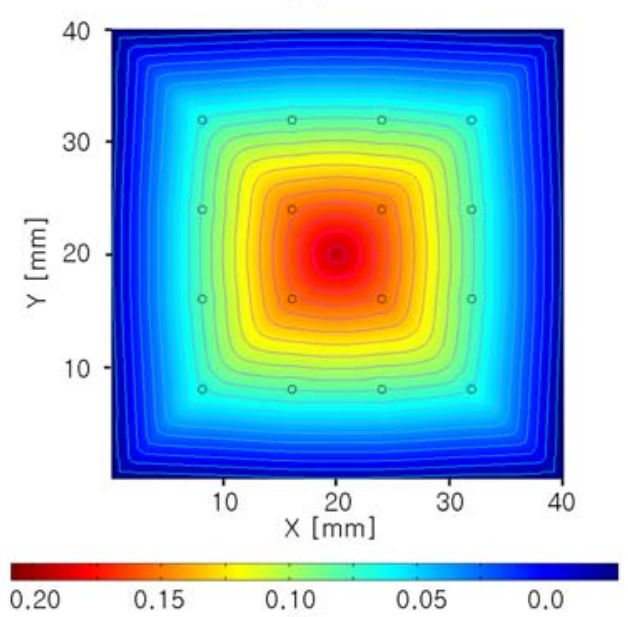

(b)

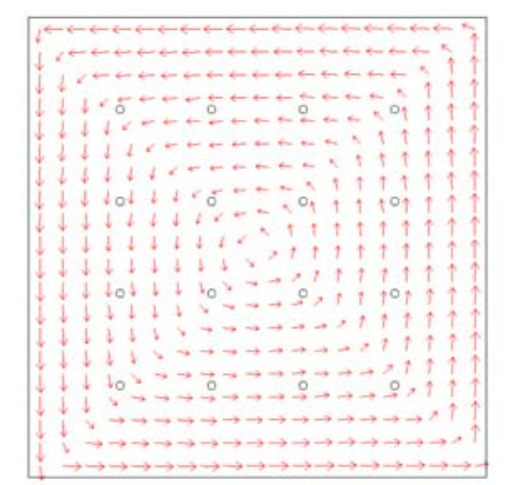

(c)

Fig. 2. Field and current distribution on the top surface of the YBCO bulk specimen: (a) measured axial field; (b) calculated axial field; and (c) calculated current distribution. because of inhomogeneity of the critical current density in the real HTS bulk.

\section{Study on Trapped Field Variation}

Effects of hole sizes and patterns on maximum trapped field capacity of an HTS bulk are investigated. The HTS bulk in the analyses is assumed to have the same properties in Table 1 except different hole sizes and patterns. Also, the external field during field cooling is assumed to be larger than the maximum trapped field, $>0.2 \mathrm{~T}$, strong enough to make the HTS bulk fully saturated. Three distinct hole sizes $-0.7,1.5$, and $3.0 \mathrm{~mm}$ - are analyzed on three different square hole patterns seen in Fig. 3. Maximum trapped field at the center, total trapped flux, and average trapped field in each pattern are compared.

\subsection{Analysis of a plain bulk without holes}

Fig. 4 shows axial field distribution and induced superconducting currents in the HTS bulk without holes. The axial field and current distributions are concentric and the peak field of $0.197 \mathrm{~T}$ was calculated at the center while the measured field was $0.198 \mathrm{~T}$.

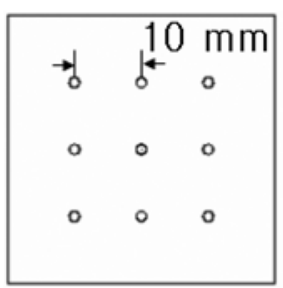

(a)

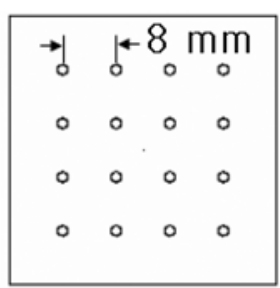

(b)

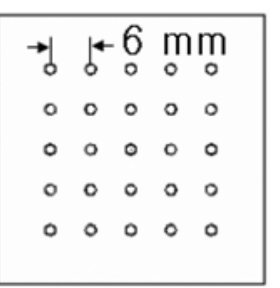

(c)
Fig. 3. Square patterns: (a) 9 holes with $10 \mathrm{~mm}$ distance; (b) 16 holes with $8 \mathrm{~mm}$; (c) 25 holes with $6 \mathrm{~mm}$. On each pattern, three holes sizes $-0.7,1.5$, and $3.0 \mathrm{~mm}-$ are analyzed.

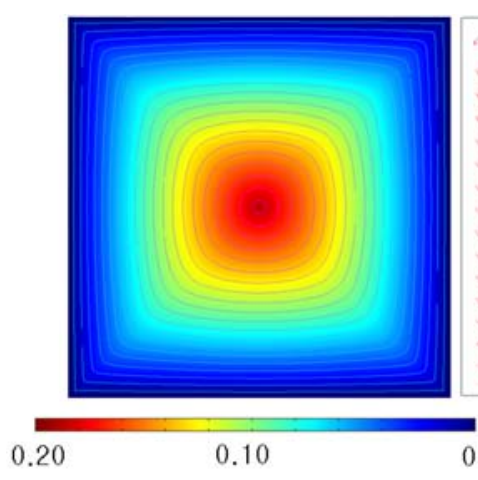

(a) Axial field

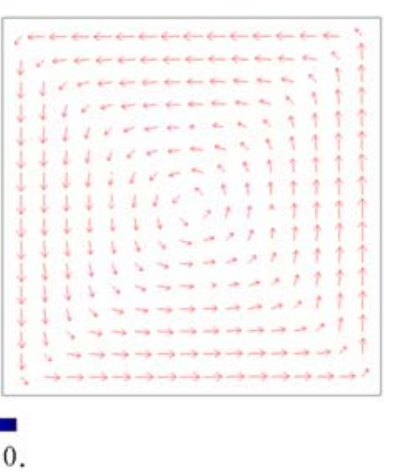

(b) Current
Fig. 4. (a) Axial field and (b) induced current distribution in the HTS bulk without a hole. Peak field of 0.197 T was calculated at the center of the top surface, which is similar to measured one, $0.198 \mathrm{~T}$. 
3.2. 9, 16, and 25 artificial holes of $0.7,1.5$, and 3.0 $\mathbf{m m}$ in diameter with square patterns

Fig. 5 presents calculation results of axial field distribution in the HTS bulk: (a) $9 \times 0.7 \mathrm{~mm}$; (b) $16 \times 0.7 \mathrm{~mm}$; (c) $25 \times 0.7 \mathrm{~mm}$; (d) $9 \times 1.5 \mathrm{~mm}$; (e) $16 \times 1.5 \mathrm{~mm}$; (f) $25 \times 1.5 \mathrm{~mm}$; (g) $9 \times 3.0 \mathrm{~mm}$; (h) $16 \times 3.0 \mathrm{~mm}$; and (i) $25 \times 3.0 \mathrm{~mm}$ holes. As the hole size increases from 0.7 to $3.0 \mathrm{~mm}$, original concentric trapped field lines in Fig. $4 \mathrm{a}$ deform more significantly. In all calculations, the peak fields were found at the center of the bulk. When the number of holes is 25 , the calculated peak fields are decreased by $1.8,8.5$, and $17 \%$ of the center field in Fig. 4a. as the hole sizes are $0.7,1.5$, and $3.0 \mathrm{~mm}$, respectively.

\subsection{Discussion}

Fig. 6 presents: (a) maximum trapped center field; (b) total trapped flux calculated by a surface integral of the axial trapped field; and (c) average trapped field calculated by the total trapped flux in Fig. 6a divided by the YBCO bulk area excluding holes.

As seen in Fig. 6a, the maximum trapped field is, in

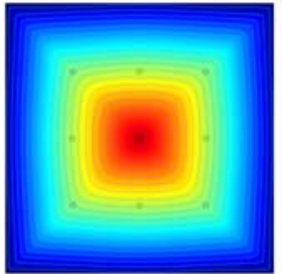

(a)

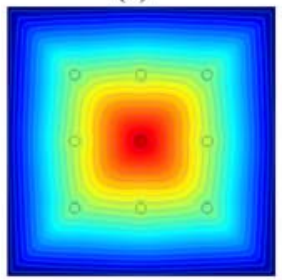

(d)

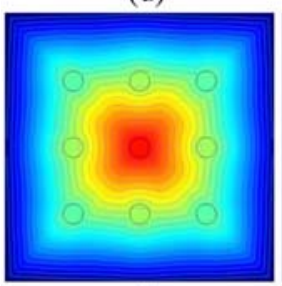

(g)

0.2

0.16

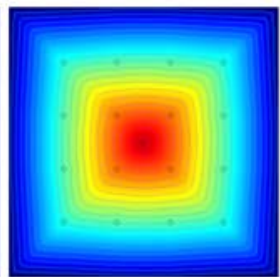

(b)

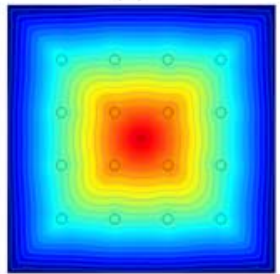

(e)

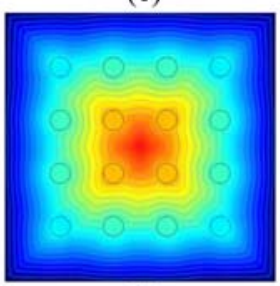

(h)

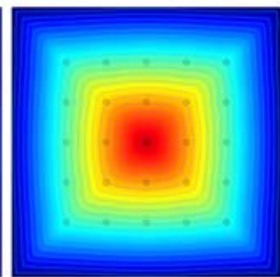

(c)

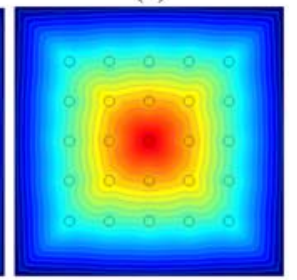

(f)

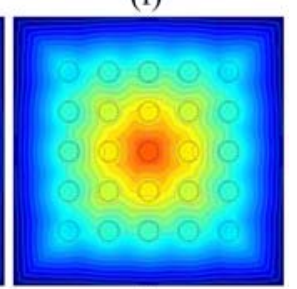

(i)
Fig. 5. Axial field distribution on the top surface of the YBCO bulk: (a) $9 \times 0.7-\mathrm{mm}$ holes with center peak field intensity of $0.1945 \mathrm{~T}$; (b) $16 \times 0.7-\mathrm{mm}$ with $0.1955 \mathrm{~T}$; (c) $25 \times 0.7-\mathrm{mm}$ with $0.1934 \mathrm{~T}$; (d) $9 \times 1.5-\mathrm{mm}$ with $0.1903 \mathrm{~T}$; (e) $16 \times 1.5-\mathrm{mm}$ with $0.1905 \mathrm{~T}$; (f) $25 \times 1.5-\mathrm{mm}$ with $0.1812 \mathrm{~T}$; (g) $9 \times 3.0-\mathrm{mm}$ with $0.1791 \mathrm{~T}$; (h) $16 \times 3.0-\mathrm{mm}$ with $0.1795 \mathrm{~T}$; and (i) $25 \times 3.0-\mathrm{mm}$ with $0.1628 \mathrm{~T}$. general, decreased as the number and size of holes are increased. However, the center field of the 16-hole case is slightly larger than that of the 9-hole case regardless of

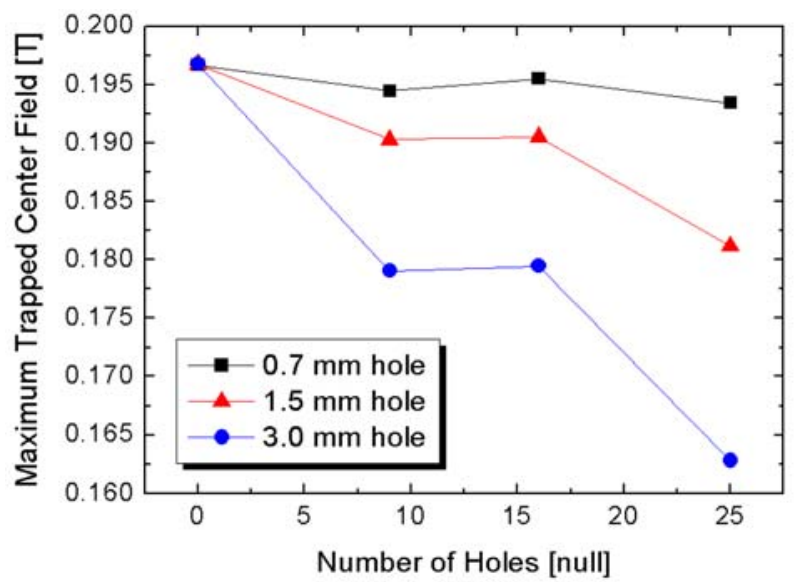

(a)

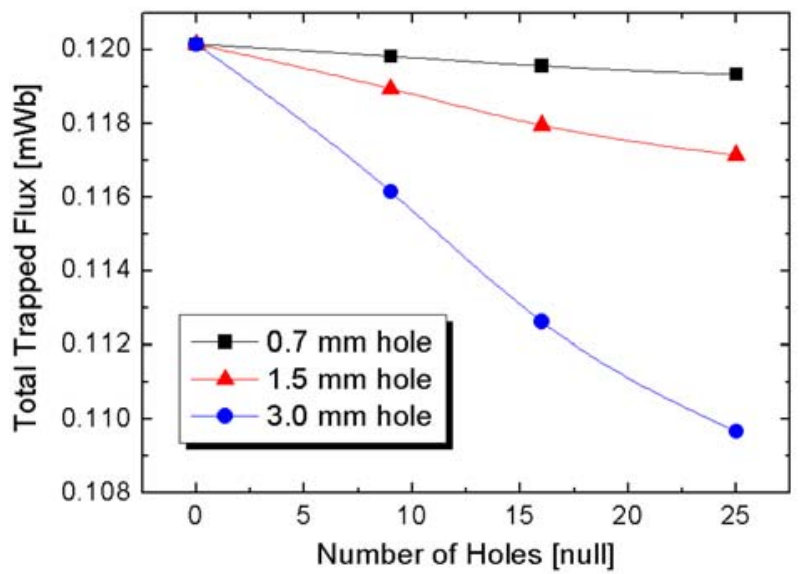

(b)

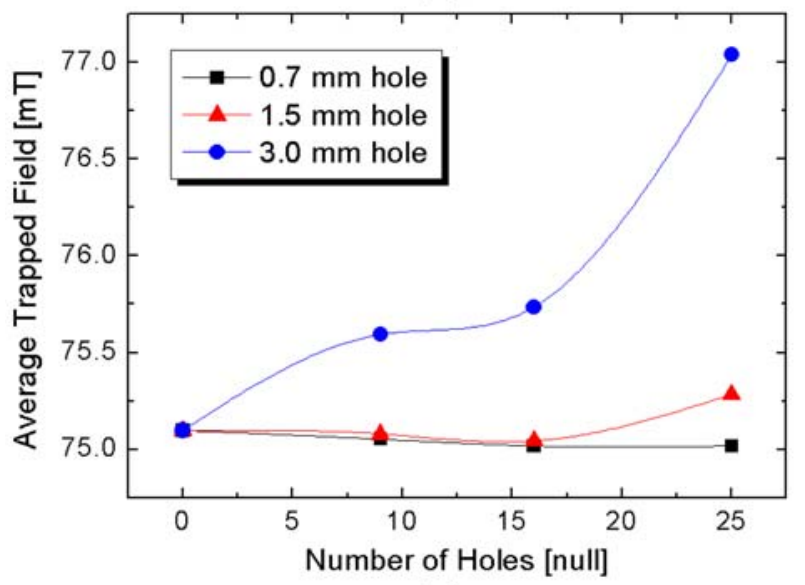

(c)

Fig. 6. Trapped field characteristics with different hole sizes and patters obtained from Fig. 4 and 5: (a) maximum trapped field at the center; (b) total trapped flux; (c) average trapped field. 


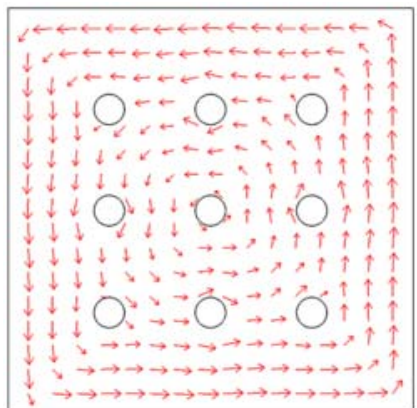

(a)

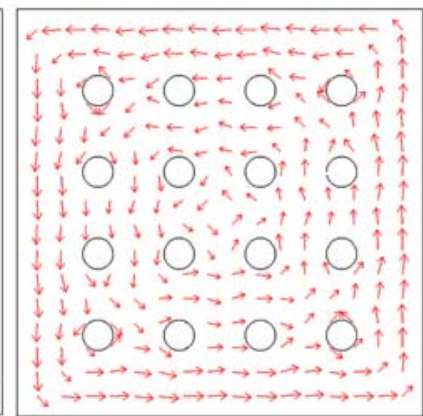

(b)
Fig. 7. Calculated current distributions: (a) $3.0 \mathrm{~mm} 9$ holes; (b) $3.0 \mathrm{~mm} 16$ holes.

hole sizes. Fig. 7 presents induced current distributions of (a) the $3.0 \mathrm{~mm}$ 9-hole case and (b) the $3.0 \mathrm{~mm}$ 16-hole. In the Fig. $7 \mathrm{a}$ an artificial hole is located at the center and no currents are induced near the hole. But, in the case $7 \mathrm{~b}$, the locally induced current near the center enhanced the peak center field. Thus, as seen in Fig. 6a, the maximum axial center field of the 16-hole case is larger than that of the 9hole in spite of the larger number of holes. This implies that the peak trapped field is dependent on a pattern of artificial holes, particularly the presence of an artificial hole at the bulk geometric center.

As seen in Fig. 6b, the total trapped flux is decreased as the number and the size of holes are increased. In a magnetic levitation system using HTS bulks, the magnitude of a levitation force is determined by a total trapped flux capacity of the bulks instead of a peak trapped field. As seen in Fig. 6c, the average trapped field is even increasing as the number and size of holes are increased chiefly owing to the weaker trapped field strength and larger $J_{c}$ in equation (5). So the primary reason of the total trapped flux reduction in Fig. $6 \mathrm{~b}$ is the effective bulk area reduction by creation of holes rather than trapped field reduction. Note that the variation of maximum trapped field and total trapped flux in the present samples are $17 \%$ and $9.0 \%$, respectively, while that of the average trapped field is only $2.5 \%$. This implies that total trapped flux is more dependent on total area of holes than their individual size and distribution. Also, note that, in a practical range of hole sizes of the present samples, 0.7$1.5 \mathrm{~mm}$, maximum trapped field, total flux density, and average trapped field were varied only within $9 \%$ up to the 25 holes.

\section{Conclusion}

A 2-D FEM using magnetic vector potential was used to analyze trapped field characteristics of an HTS bulk with scattered artificial holes. The validity of the suggested method was verified by comparison between calculation and measurement of the trapped field distribution in an HTS bulk with 16 artificial holes. And, effects of various artificial holes on trapped field variations were investigated using the suggested method. Based on the results obtained in this research, we may conclude that:

- The suggested numerical method may be useful to estimate maximum trapped field capacity of an HTS bulk with various artificial holes.

- The peak trapped field at the center of an HTS bulk is affected by the size and location of artificial holes.

- The total trapped flux, however, is more dependent on the overall area of holes than their size and location.

- In a practical range of hole sizes, $0.7-1.5 \mathrm{~mm}$ in diameter, maximum trapped field, total flux density, and average trapped field were estimated to vary within $9 \%$ up to the 25 holes.

- For more accurate calculation, 3-D analysis will be required to take finite length of the rectangular bulks into consideration.

\section{Acknowledgement}

This work was supported by the Power Generation \& Electricity Delivery of the Korea Institute of Energy Technology Evaluation and Planning (KETEP) grant funded by the Korean Ministry of Knowledge Economy.

\section{References}

[1] M. Tomita and M. Murakami, Nature 421, 517 (2003).

[2] S. Haindl, F. Hengstberger, H. W. Weber, S. Meslin, J. Noudem, and X. Chaud, Supercond. Sci. Technol. 19, 108 (2006).

[3] P. Diko, S. Kracunovska, L. Ceniga, J. Bierlich, M. Zeisberger, and W. Gawalek, Supercond. Sci. Technol. 18, 1400 (2005).

[4] N. Lee, G. E. Jang, C. Kim, T. Sung, Y. Han, and S. Jung, Physica C 463, 320 (2007).

[5] E. Bartolome, X. Granados, T. Obrados, E. S. Reddy, and S. Kracunovska, IEEE Trans. Appl. Supercond. 15, 2775 (2005).

[6] H. Fujishiro, T. Naito, D. Furuta, and K. Kakehata, Physica C 470, 1181 (2010).

[7] S. Hahn, J. H. Kim, C. Koh, and S. Hahn, IEEE Trans. Appl. Supercond 11, 1996 (2001).

[8] H. Ueda, M. Iwamoto, Y. Kudo, and A. Ishiyama, Electrical Engineering in Japan 159, 44 (2007).

[9] R. Gonzalez-Arrabal, M. Eisterer, and H. W. Weber, J. Appl. Phys. 93, 4734 (2004).

[10] M. Tsuchimoto and T. Honma, IEEE Trans. Appl. Supercond. 4, 211 (1994). 